\title{
La Pitié (1803) et La Conversation (1812) de Jacques Delille par rapport aux recherches sur la voix humaine et la parole
}

\author{
Jacques Delille's La Pitié (1803) and La Conversation (1812) \\ in the light of scientific theories on human voice and speech
}

\author{
Michał Bajer \\ Uniwersytet Szczeciński \\ michal.bajer@usz.edu.pl
}

\begin{abstract}
The studies of Jacques Delille's work put an emphasis on the relations between a group of poetic texts (L'homme des champs, L'Imagination, Les Trois Règnes de la nature) and their scientific intertexts. The aim of this paper is to study the scientific connotations of lesser known poems, stressing the problem of the physiological and anatomical reflections on the human voice and the speech. Is there a relation between Delille's observations on the voice (La Pitié, p. 40, 151-152 ; La Conversation, p. 141-142, 146-147) and the terminology used by physicians or philosophers of his time? What is the impact of Rousseau's Essai sur l'origine des langues on Delille? Is it inspiring to use the medical distinction between the physiology and the pathology to understand Delilles's conception of the human voice?
\end{abstract}

Keywords: Jacques Delille, Nicolas Antoine Taunay, audition, attention, articulation, Joseph-Marie de Gérando, Jean Marc Gaspard Itard, conversation's rules

À partir de 1800, Jacques Delille fait alterner les «épopées de la nature » (L'Homme des champs, 1800 ; Les Trois Règnes de la nature, 1808) et les poèmes centrés sur les interactions humaines, dont les exemples les plus notables sont $L a$ Pitié et La Conversation. Les deux types d'œuvres s'entrelacent dans la production de l'auteur au point de fusionner plus d'une fois. S'il est certain que les concepts d'ordre éthique façonnent chez Delille l'étude et la description de la nature, je propose d'interroger le rôle des catégories empruntées aux sciences du vivant dans 
les textes dédiés à la problématique morale et sociale. Réinterprétés à la lumière des travaux plus anciens (Guitton, 1976) et récents (Stalnaker, 2010 ; Marchal \& Wanlin, 2014 ; Marchal, Louâpre \& Pierssens, 2014), les procédés linguistiques, littéraires et textuels associés chez Delille à la poétisation des phénomènes biologiques ne sont pas un simple ornement du discours, mais ils constituent un outil herméneutique puissant, permettant d'explorer les facettes multiples des engagements sociaux et religieux de l'homme. Parmi de nombreux problèmes relatifs à la biologie et la médecine, les poèmes delilliens sur les mœurs attribuent une place particulière aux questions de la communication envisagée tant dans ses fondements anatomiques et physiologiques que du point de vue philosophique. Abordée déjà dans Les Trois Règnes de la nature, la production et la perception de la voix articulée jouent un rôle crucial dans La Conversation. Il en est de même dans La Pitié, où les inflexions de la voix humaine apparaissent comme facteur important dans l'expression et la stimulation de l'affect éponyme - mis à part la récurrence de la formule métaphorique, teintée d'allégorie, « la voix de la pitié » (« La Pitié dans les cœurs fait entendre sa voix » [Delille, $1803^{1}$, p. 81]).

L'histoire de la réflexion scientifique sur la production et la perception de la parole constitue une trame importante dans des travaux à intérêt général (Foucault, Derrida, Moravia), tout en faisant l'objet des études plus ciblées (Neboit-Mombet, 2001 ; Jamain, 2004 ; Steuckardt \& Thorel, 2017). Le moment fort de cette histoire était l'intervention de Cabanis, qui - sur les traces de Locke, Condillac, Bonnet et Hélvétius - plaça l'activité du cerveau au centre de la recherche philosophique et médicale (Clauzade, 2008 ; Williams, 1994). Les questions de la voix et de la parole ont été approfondies ensuite par un groupe d'auteurs dont les textes seront évoqués de façon ponctuelle dans la présente étude. Sans offrir une vision d'ensemble, je proposerai donc une série de rapprochements entre la production delillienne et la pensée scientifique de son époque. Tout hypothétiques qu'ils soient à ce stade de la recherche, ces rapprochements semblent plausibles à la lumière des travaux sur la biographie intellectuelle de Delille et des membres de son entourage. Les développements sur la voix humaine et la parole évoqués plus loin émanent, pour la plupart, des auteurs liés aux institutions cruciales pour l'organisation de la vie scientifique après la Révolution (notamment à l'Institut de France).

Sur le fond des parallélismes biographiques et sociologiques, il devient possible d'indiquer quelques traits, déterminant la problématisation du phénomène de la voix par des chercheurs partageant un certain nombre d'expériences communes. Cette approche supra-individuelle peut être illustrée - à titre d'exemple - par la citation tirée d'Éloge de Félix Vicq-d'Azyr de Jacques-Louis Moreau, dédié à Cuvier :

La respiration n'est pas seulement un des premiers moyens de la vie; elle sert encore à établir une correspondance intime entre les animaux. L'air expiré se convertit en sons

\footnotetext{
${ }^{1}$ Désormais abrégé en $P$.
} 
indéfiniment variés, devient ainsi l'élément des voix diverses, et rapproche la plupart des êtres animés par un langage sans lequel la nature silencieuse semblerait plongée dans un sommeil éternel (Moreau, 1797-1798, p. 24).

Tout d'abord, en faisant l'écho à certains éléments de la tradition des Lumières (que Vicq-d'Azyr perpétuait et reconfigurait), ce passage situe le problème de la voix dans le contexte des autres fonctions physiologiques. Ensuite, par le fait de mettre en avant les parallélismes entre de différents animaux, il annonce la domination de l'anatomie comparée dans les sciences du vivant des années à venir. Enfin, l'image métaphorique de la nature ensommeillée suggère une ouverture vers les considérations d'ordre moral qui - malgré la spécialisation poussée des sciences de la nature durant la période postrévolutionnaire - reviendra quasi régulièrement dans des traités des médecins et des naturalistes lorsqu'ils aborderont les questions de la communication. Sans imposer l'unité quelconque aux travaux des chercheurs différents, ces trois traits orientaient une partie importante des débats menés autour des institutions dont les activités interféraient avec celles de Jacques Delille et de ses contemporains. À tout ceci s'ajoutèrent d'importantes élaborations proposées dans le domaine des arts de la voix (l'éloquence, le chant, la déclamation, la lecture à haute voix etc.) dont une petite partie j'évoquerai au cours de l'article ${ }^{2}$.

Voici - en grand raccourci - le contexte intellectuel dans lequel viennent se placer La Pitié et La Conversation. Même si la poétisation des phénomènes de la parole se fait chez Delille en dehors des rigueurs méthodologiques de la science de son époque, le détour par les catégories scientifiques permettra, peut-être, d'explorer quelques pistes qui mènent vers la perception de l'œuvre delillienne dans son unité.

\section{LA PRODUCTION DE LA PAROLE}

Dans la description d'un homme bruyant, Delille utilise, entre autres, les passages $\mathrm{du}$ poème Le Conversazioni de Clemente Bondi (Delille, $1812^{3}$, p. 220-223). Il s'approprie le texte italien non seulement à travers la traduction, mais aussi par une nouvelle division de la matière. Les idées lancées d'une seule traite par Bondi deviennent dans La Conversation des catégories séparées. Dans le texte français 22 vers sont consacrés spécifiquement à la formation de la voix, contre 8 seulement dans le poème italien. Les procédures d'adaptation montrent donc le vif effort de Delille à faire de l'articulation humaine un objet poétique autonome, pouvant

\footnotetext{
${ }^{2}$ L'ouverture dans cette direction a été suggérée par Delille lui-même, qui dans les notes accompagnant sa traduction d'Enéide, défend l'idée de l'harmonie imitative, qu'il reconnaît, notamment, dans l'emploi des allitérations et des structures de versification inattendues, avant de mentionner l'effet de ces procédés dans la déclamation - en rapprochant par ce geste la réflexion poétologique de la théorie de l'art scénique (Virgile, 1804a, p. 208-210 ; voir aussi Virgile, 1804b, p. 272, 400-401).

${ }^{3}$ Désormais abrégé en $C$.
} 
rivaliser avec la topique traditionnelle des traités sur la sociabilité. En même temps, Delille remplace les expressions stéréotypées, utilisées par Bondi dans ses remarques sur la production de la parole («grida con alta voce» etc.), par une terminologie beaucoup plus précise ( « rauques accents ", " échelle vocale », "l'organe humain » etc. [C, p. 141-142]). Le fragment culmine dans une série de conseils techniques sur la prononciation adaptée à l'art de la conversation :

Il faut, en conversant, qu'un heureux artifice

De l'échelle vocale étudiant les tons,

Adoucisse à propos ou renforce les sons.

L'organe humain ne veut ni roideur, ni mollesse :

Trop faible il nous échappe, et trop fort il nous blesse [...]. (C, p. 141).

Quel est le fondement théorique des conceptions exposées ici et dans d'autres textes de Delille traitant de la voix humaine ? La question est difficile, dans la mesure où - contrairement à un grand nombre d'autres passages - les développements sur la production de la parole ne contiennent de références bibliographiques explicites ni dans les vers, ni dans les notes. Le silence des commentateurs peut être expliqué, entre autres, par la clarté relative de l'exposé delillien dans les fragments en question. En même temps, l'absence de références explicites semble significative en elle-même. Dans le réaménagement des institutions scientifiques de la France post-révolutionnaire (Marchal, 2014, p. 91-106; Wanlin, 2014, p. 3 ; Corsi, 2018, p. 13-19), plusieurs théories de la langue héritées du siècle précédent pouvaient sembler suspectes. Qu'elles aient émané des sciences naturelles taxées de matérialisme antireligieux, qu'elles soient venues de la philosophie de la nature allemande, ou qu'elles se laissent rattacher à l'Essai sur l'origine des langues de Rousseau, elles avaient de quoi contrarier les gardiens de la nouvelle hiérarchie des autorités scientifiques ${ }^{4}$. Ce fait peut expliquer à la fois le caractère plutôt général des développements delilliens sur l'articulation et l'absence de toute mise en contexte bibliographique précise. La circonspection de l'auteur à engager le dialogue avec l'héritage des Lumières frappe particulièrement dans Les Trois Règnes..., où l'on pourrait s'attendre à une allusion quelconque à l'origine de la parole. En effet, le passage sur la langue s'ouvre par une mise en perspective historique (« Mais ces arts bienfaisants que l'instinct fit éclore,/ Dans leur obscur berceau semblaient languir encore » [Delille, 1808, p. 261]) qui reste néanmoins à l'état d'esquisse, coupée court par le parallèle entre l'homme et l'animal, développé en dehors de tout contexte chronologique.

Dans cette stratégie, somme toute négative, visant à éviter les références scientifiques précises, un fragment semble trahir les traces d'une influence, dont la source est passée sous le silence. Dans la phrase déjà citée de La Conversation, Delille

\footnotetext{
${ }^{4}$ « [S]elf-censorship was the only measure naturalists could take to rehabilitate scientific endeavors in the eyes of reactionary authorities $[\ldots] »($ Corsi, 2018, p. 17).
} 
encourage son lecteur à étudier les tons de «l'échelle vocale » $(C, \mathrm{p}$. 141), en reprenant une expression technique fréquente tant dans la critique musicale (voir p. ex. Castil-Blaze, 1820, p. 257) que dans les textes liant la problématique artistique et médicale (Colombat, 1838, p. 326). À travers cette expression, Delille introduit dans son poème l'écho des discussions sur le mécanisme de l'intonation humaine, signalées déjà dans l'Encyclopédie (Anonyme, 1765, p. 428) et chez Antoine Court de Gébelin, auteur de transition, liant la pensée préscientifique avec la philosophie des Idéologues ${ }^{5}$ : «Puisque l'instrument vocal, considéré dans ses sons, est un instrument à vent, il faut nécessairement qu'il produise une octave [...]. Et puisqu'étant considéré dans ses intonations, il est un instrument à touches, il n'est pas étonnant qu'on y remarque encore l'empreinte de la même harmonie » (1775, p. 127). Si l'approche schématique de Court de Gébelin a été rejetée par les générations suivantes, les textes plus tardifs font état des controverses anciennes, tout en témoignant d'un désarroi face à la difficulté de la question : «Tous ces [divers] auteurs diffèrent sur la question de savoir à quel instrument doit être assimilé le larynx pour créer les différents tons qui constituent l'échelle vocale » (Potton, 1843, p. 6).

D’une façon générale, les métaphores et comparaisons musicales sont peu présentes dans La Conversation. Ce procédé permet de protéger le statut du phénomène éponyme comme discipline à part, en prévenant sa confusion avec d'autres arts utilisant la voix. Dans ce contexte, l'expression «échelle vocale » fait figure d'exception confirmant la règle, également parce qu'elle ramène un écho discret de la théorie rousseauiste de la langue («[...] les vers, les chants, la parole, ont une origine commune » [Rousseau, 1969, p. 529]). Ainsi assure-t-elle la présence minimale à l'élément auquel une place plus importante a été délibérément refusée ${ }^{6}$.

Malgré l'absence des références explicites à des textes scientifiques précis, un certain groupe de catégories basiques lie les poèmes de Delille aux recherches contemporaines sur la voix et la parole. Dans La Pitié, le mot «accent» désigne notamment l'intonation trahissant une forte émotion (p. ex. « accent douloureux » [P, p. 44]), dans La Conversation - l'intonation chargée d'un trait éthique (p. ex. « accents pleins de noblesse » [C, p. 40]). Dans ce dernier ouvrage, le mot «ton» intervient également pour caractériser la plasticité de la voix, capable d'exprimer l'attitude morale et sociale de l'homme ou de la femme (p. ex. " ton guerrier» [C, p. 45]). Ailleurs encore, l' « accent» renvoie aux inflexions propres à la voix humaine en général (« accent humain $»[P, \mathrm{p}$. 151) et à une façon de parler vicieuse du point de vue esthétique :

\footnotetext{
${ }^{5}$ Sur Court de Gébelin, voir Mercier-Faivre (1999, p. 19-75).

${ }^{6}$ En ce qui concerne plus précisément le rapport à Rousseau - évoqué plusieurs fois dans les textes de Delille (voir p. ex. $C$, p. 184) - plusieurs facteurs pouvaient retenir le poète de laisser à cet auteur une place plus éminente dans La Conversation, y compris l'attitude généralement hostile de Rousseau par rapport aux manifestations formelles de la sociabilité.
} 
Surtout il se défend des sons durs que hasarde

Des parleurs mal instruits la nation criarde ;

Dans les clubs ébranlés par leurs rauques accents

Il laisse s'enrouer leurs gosiers glapissants (C, p. 141).

À cette caricature s'oppose la formule «bon ton » (voir p. ex. $C$, p. 101). Finalement, Delille place une allusion explicite à la hauteur des sons, où le mot «ton » apparaît au sens harmonique (l'expression «l'échelle vocale », déjà citée [C, p. 141]).

D'un côté, le texte reflète donc la flexibilité sémantique propre à l'usage courant. Selon les dictionnaires de l'époque, le mot «ton » désigne soit la hauteur du son, soit sa charge émotionnelle, parfois liée au volume (dans l'expression «baisser le ton »). Plusieurs idées se confondent chez Delille dans la présentation raccourcie des problèmes qui chez Cuvier, par exemple, donnaient lieu à des distinctions scrupuleuses : les Leçons d'anatomie comparée séparent le ton (« les divers degrés de grave et d'aigu ») et l'intensité du son (Cuvier, 1805b, p. 445).

D'un autre côté cependant, le texte poétique reflète un élément important de l'exposé de Cuvier. Leçons d'anatomie comparée distinguent la sonorité de la voix, propre aux voyelles, et l'articulation au sens étroit, propre aux consonnes (Cuvier, 1805 b, p. 446). Or, le troisième chant de La Conversation traite les deux phénomènes dans des passages à part. La caricature déjà évoquée d'un criard $(C, \mathrm{p} .141-142)$ concerne le volume que la voix humaine est capable de contrôler notamment en prononçant les voyelles. Plus tard, Delille dresse le portrait d'un homme atteint des troubles de la parole :

La langue qui, dans le palais,

Cherchant des mots qui n'arrivent jamais,

Semble, en balbutiant la plus belle pensée,

Du filet de l'enfance encore embarrassée,

Et dont le bégaiement, consolant le muet,

À chaque son qu'elle tâche d'émettre,

Tourmente en vain tout l'alphabet

Et lutte contre chaque lettre ( $C$, p. 146).

Même s'il est question ici de "toutes les lettres d'alphabet», le texte renvoie explicitement au bégaiement que les médecins de l'époque liaient à la prononciation des consonnes (Voisin, 1821, p. 30).

\section{LA PERCEPTION DE LA PAROLE}

À côté de la production de la parole, c'est également sa réception qui semble intéresser Delille dans les poèmes centrés sur les interactions humaines. La continuation du passage déjà cité sur l'interlocuteur criard apporte un tableau suivant : 
On se plaît à causer avec ses bons amis ;

Mais quand leur voix trop forte à l'orage est pareille,

Leur amitié devient un tourment : notre oreille

appelle la parole $\mathrm{e}^{7}$ et repousse les cris $(C$, p. 141-142).

La versification rend visible (et audible) le phénomène dont il est explicitement question dans cet extrait. Les positions du substantif en contre-rejet et du verbe en rejet mettent en exergue le mot « appelle», comme pour souligner l'effort nécessaire à l'accomplissement de cette action ${ }^{8}$. On remarquera de surcroît que le passage cité reprend une formule présente déjà avant, à l'occasion de la caricature d'un homme éprouvant des difficultés à laisser parler quelqu'un d'autre que lui-même. En présence d'une personne exprimant ses opinions, il: "Accélère les tours [de l'interlocuteur], diligente les mots,/ Vient au-devant de mes propres propos,/ A p pelle la p a rol $\mathrm{e}^{9}$, accouche la pensée » $(C, \mathrm{p}$. 64). Très curieusement, la perception du discours considérée à l'échelle abstraite $(C, \mathrm{p} .142)$ est donc décrite à l'aide de la même expression qui sert à caractériser le mauvais auditeur. Le point commun entre l'oreille humaine en général et le discoureur importun est une forme d'énergie qu'ils mettent tous deux dans l'exercice de leurs fonctions respectives. Comme le bavard impatient, l'organe de l'ouïe n'est pas un récepteur passif des sonorités mais il « appelle » certaines d'entre elles, pour en « repousser » d'autres.

Depuis l'Encyclopédie jusqu'au Premier Empire, un minimalisme éclairé et méthodiquement rigoureux domine les exposés sur la relation entre l'ouïe et la faculté de parler. On répète que malgré un grand nombre de faits établis dans le domaine de l'activité des organes articulatoires et du système auditif, la perception du discours - phénomène situé au carrefour des compétences diverses - reste particulièrement difficile à expliquer («[...] d'où naît l'accord qu'il y a entre l'ouïe \& la parole ? [...] [O]n n'est pas encore d'accord des effets de ces communications » [Jaucourt, 1765, p. 618]). Dans sa leçon De l'organe de l'ouïe ou de l'oreille, Cuvier (1805a, p. 449-50) souligne la capacité de l'oreille des mammifères à percevoir les nuances produites par la voix humaine, tout en constatant finalement le peu de certitude quant au détail de la corrélation entre le système auditif et son objet (voir aussi Savary, 1812, p. 456). Malgré les mystères entourant les détails de l'audition de la parole, son lien avec les activités du cerveau paraît évident, et ceci bien avant l'étape de l'analyse du contenu du discours, située en aval du processus de sa perception, mais aussi en amont : le cerveau se voit attribuer une part active dans la configuration des sonorités servant de véhicule à l'échange verbal. Le rôle important est assigné ici à l'attention, étudiée sous l'angle de la philosophie par Gérando :

\footnotetext{
${ }^{7}$ C'est moi qui souligne.

${ }^{8}$ Rappelons que Delille voit dans les effets de versification la réalisation de 1' «harmonie imitative » (Virgile, 1804a, p. 210).

${ }^{9} \mathrm{C}$ 'est moi qui souligne.
} 
Il ne faut pas oublier que l'attention est toujours une condition nécessaire pour la liaison des idées. Ainsi, les signes lui doivent aussi une partie de leur vertu, et parmi les sensations simultanées, successives ou analogues, celles-là seules deviendront signes, qui auront été suffisamment remarquées (Gérando, 1799, p. 68).

Au tournant du XVIII ${ }^{\mathrm{e}}$ et $\mathrm{XIX}^{\mathrm{e}}$ siècles, l'étude de l'attention était suffisamment à la mode, pour que le titre de précurseur dans ce domaine de recherche soit l'objet d'une rivalité ${ }^{10}$.

En suivant la direction indiquée par Gérando, Delille valorise l'attention comme auxiliaire nécessaire de toute "liaison des idées» dans l'acte de communication réussi. Les troubles de communication sont pour lui, dans beaucoup de cas, les dérèglements de l'attention. Le bavard compulsif manifeste une sorte d'attention grotesque («tyrannie attentive» $[C$, p. 65]), tournée non pas vers l'autre, mais vers soi. Le «défiant», son reflet renversé, toujours alerte, soupçonne une marque de désapprobation derrière chaque geste de son interlocuteur ("inquiète folie » [C, p. 109]). Il souffre des effets de l'attention délirante qui - d'après l'expression proposée par de Gérando - remarque chaque sensation pour en faire un signe. Dans le portrait de l'interlocuteur idéal, l'éloge de l'« attention encourageante » $(C$, p. 150) indique la qualité de l'écoute comme facteur principal du lien social. Finalement, l'inattention contrôlée devient la défense ultime contre un discoureur importun ( « Vous avez, en effet, parlé, ne vous déplaise,/ Assez longtemps, mais je n'écoutais pas », $C$, p. 70). L'attention - comme la voix, la mimique etc. - peut donc être entraînée, activée et désactivée au gré de l'individu et en fonction des circonstances. Ce fait accentue son lien avec le corps, dont la discipline - résidant au centre de tout processus de socialisation - fournit une des bases à la théorie delillienne de la conversation.

Pris globalement, ce traitement du problème de l'attention semble symptomatique pour l'époque de transition où la science moderne voisinait encore avec les approches préscientifiques et les Lumières tardives rencontraient les impulsions plus modernes (Marchal, 2014, p. 103-105 ; Wanlin, 2014, p. 2-3). Or, malgré son importance pour certains, la réflexion sur l'attention reflète la tendance générale des sciences du vivant dans la période étudiée, c'est à dire leur marche continue vers la spécialisation de plus en plus poussée. Charles-Louis Dumas (1800a, p. 488-489) associa la problématique de l'attention à l'analyse des interactions humaines qui, quant à elles, « appartiennent pour le moins à la métaphysique autant qu'à la physiologie » (Dumas, 1800b, p. 599). Après quelques questions préliminaires, Cuvier décida d'exclure du domaine de la physiologie le groupe de sujets dont la présence dans la médecine remontait à l'antiquité (le fonctionnement de l'imagination, de

\footnotetext{
${ }^{10}$ Voir les déclarations de Dugald Stewart et leur rectification par le traducteur, Pierre Prévost, dans la note ajoutée au texte français (Stewart, 1808, p. 165).
} 
l'attention etc.). La réflexion complexe sur les sens internes se voit déléguée vers la métaphysique, seuls les mouvements corporels correspondant aux idées sont étudiés dans le traité (Cuvier, 1805a, p. 110-111). On comprend donc que sur le fond de la rigueur terminologique, promue notamment par Cuvier, l'intérêt pour les mécanismes de l'attention doit être considéré comme problématique à la lumière des sciences du vivant modernes. En effet, le traitement delillien de cette question à travers une série de caricatures dans La Conversation mobilise plutôt des catégories préscientifiques : il fait d'évidence écho aux pathologies du caractère, étudiées sur un fond galénique par les écrivains de l'âge classique. Dans La Pitié, l'analyse de la perception transformée sous l'effet de la terreur ${ }^{11}$ renoue avec la réinterprétation de la médecine grecque par les libertins de la première moitié du XVII ${ }^{\mathrm{e}}$ siècle, en faisant penser par exemple aux Stances de Théophile de Viau ${ }^{12}$. Néanmoins, tout en apportant une poétisation du monde en dehors de la rigueur des divisions scientifiques modernes, l'approche delillienne tourne-t-elle définitivement le dos aux préoccupations des chercheurs de son époque?

$\mathrm{Au}$ début du $\mathrm{XIX}^{\mathrm{e}}$ siècle un groupe de disciplines ne peut, par nécessité, s'arrêter à des réponses partielles, pour sûres et vérifiables qu'elles soient. Ces disciplines sont représentées par les thérapeutes ainsi que par les précurseurs de l'éducation spécialisée et de la psychopédagogie (Gineste, 1989, p. 115), mettant en pratique le savoir scientifique dans le but d'entraîner l'amélioration de l'état d'un individu confié à leurs soins. Tant ces médecins que ces éducateurs s'intéressent aux problèmes relatifs à l'incapacité de former la parole. Tout en respectant la rigueur méthodologique de leur époque, ils sont obligés d'explorer des pistes inconnues. Ils ne le font pas en anatomiste ou physiologiste se livrant à l'observation et à l'expérimentation, mais dans l'action et dans l'échange avec un individu particulier. Le souci pédagogique et les besoins spécifiques de son élève conduisent JeanMarc-Gaspard Itard, le plus célèbre d'entre eux, à explorer d'une façon à la fois théorique et pratique le lien entre l'audition et la parole. En partant d'une série de questions et d'observations, le médecin imagine, souvent ad hoc, un programme d'expériences visant, en définitive, à sensibiliser Victor d'abord aux sons, ensuite aux paroles (Itard, 1894, p. 27-37). Ses expériences installent un va-et-vient continuel entre les principes de la physiologie et la problématique de la sociabilité. Dans le domaine des sciences du vivant c'est donc la pratique thérapeutique et pédagogique qui présente une analogie structurelle inattendue avec le projet artistique de

\footnotetext{
${ }^{11}$ « Le char affreux, roulant dans un profond silence,/ Ce char, qui, plus terrible entendu de moins près, / Du crime en s'éloignant avance les apprêts » $(P$, p. 104) ; « On vient, le verrou crie, on l'entraîne ; je tremble $»(P$, p. 108).

${ }^{12}$ « Mais quand l'arrêt sanglant a résolu sa peine/ Et qu'il voit le bourreau [...]/ Il n'a goutte de sang qui ne soit lors glacée,/ Son âme est dans les fers ;/ L'image du gibet lui monte à la pensée,/ Et l'effroi des Enfers » (Viau, 1941, p. 58).
} 
Delille, qui - dans des poèmes dits «de nature » - indiqua l'intérêt pour le détail scientifique comme manifestation d'une sensibilité nouvelle, façonnant l'ensemble des expériences humaines. Malgré toutes les différences qui les séparent, tant les médecins/éducateurs que le poète opèrent une synthèse des disciplines, rejetée ouvertement par les autorités de la génération de Cuvier (Corsi, 2018, p. 17-18).

Les disciplines pratiques étudient l'influence des pathologies du corps ainsi que celle du milieu sensoriel et social sur l'aptitude à comprendre et produire la parole. Or, c'est un sujet important pour Delille. La Pitié explore - à l'exemple de l'auteur lui-même - la perception de la voix humaine par une personne non voyante $(P$, p. 40$)$. Le quatrième chant du même poème contient en plus l'image d'un exilé qui découvre un hameau français perdu dans la forêt exotique. Le texte offre l'élaboration de plusieurs idées importantes pour les recherches sur la parole. Delille présente l'effort de l'individu exposé soudainement au contact de la voix humaine (« un chant français » $[P, \mathrm{p} .151])$ après une longue période de privation :

Il s'arrête enchanté : tout-à-coup, ô merveille!

Les sons d'un chant français ont frappé son oreille.

Trois fois, plein de surprise, il écoute ; et trois fois

Arrive jusqu'à lui cette touchante voix.

Son cœur bat de plaisir, ses yeux versent des larmes :

Jamais accent humain n'eut pour lui tant de charmes !

« Des Français sont ici », s'écria-t-il soudain;

« Je verrai des Français ! », Il dit, suit son chemin ( $P$, p. 151-152).

Le retour du même morphème ("enchanté », "chant») et l'homophonie ( «sont », «son ») traduisent l'idée de l'intériorisation du phénomène perçu par le sujet de la perception. Après les hésitations initiales, engageant l'activité de l'attention au sens indiqué par Gérando («Trois fois, plein de surprise, il écoute »), l'identification des données sensorielles conduit aux changements corporels (les pleurs) et aboutit à une explosion : l'exclamation. Comme le tout premier cri humain chez Rousseau (1969, p. 506), la réaction de l'individu ayant perdu l'habitude de parler est la nomination déclenchée par l'entrée inattendue des autres dans son champ de perception : «Des Français sont ici » ( $P$, p. 152). L'analyse rousseauiste de l'origine de la parole place dans une succession chronologique, d'abord l'apparition du terme figuré (appeler les hommes " géants »), ensuite la création du terme propre («les gens »). La Pitié offre une continuation surprenante du mécanisme reconstruit par Rousseau. Dans une substitution justifiée du point de vue des divisions logiques, au lieu du nom du genre ( « les gens » chez Rousseau) Delille place le nom de l'espèce ( « Français »).

Sur ce fond, la phrase «Jamais accent humain n'eut pour lui tant de charmes!» $(P$, p. 151) élargit la signification du terme « accent » qui - dans de nombreux textes de l'époque - sert à désigner une particularité locale (Du Marsais, 1751, p. 63). 
Deux identifications collectives, chères aux hommes et femmes des Lumières, se côtoient, offrant encore une fois un aperçu synthétique de la situation de Delille dans l'histoire des idées en Europe moderne. L'accord harmonieux de l'universalisme («accent humain ») et du particularisme national («chant français ») fait de ce passage un lieu de rencontre symbolique de Voltaire avec Herder.

\section{CONCLUSION}

Même si l'on prend en considération toutes les licences permises au poème didactique des Lumières tardives, le traitement delillien des questions de la voix et de la parole dans La Pitié et La Conversation n'atteint pas la cohérence propre à la poétisation d'autres idées scientifiques dans L'Homme des champs ou Les Trois Règnes... Ceci dit, l'étude des fonctions de l'imaginaire scientifique dans les poèmes sur la vie sociale et la morale semble prometteuse, dans la mesure où elle permet de ramener à un dénominateur commun les textes les plus lus de l'auteur (L'Homme des champs etc.) et les ouvrages rarement revisités par la critique moderne. Montrer la relation de ces différentes œuvres avec des intertextes du même type (travaux scientifiques) permet d'entamer une réflexion sur l'unité de la production du poète qui, dès ses premiers essais littéraires, privilégia la science et l'étude des mœurs en tant que deux champs d'exploration, assignés au poème didactique. Loin d'en donner une approche exhaustive, le présent article signale une application possible de ce modèle de lecture. Deux conclusions générales peuvent être avancées.

Premièrement - face à l'absence de références bibliographiques précises - il faut garder une prudence extrême dans la recherche des sources des conceptions delilliennes concernant la voix et la parole. D'un côté, on y remarque la reprise des vacillements sémantiques propres à l'usage courant. En plus - vu la tendance générale de l'époque - le fait de passer sous un silence total l'origine de la parole permet de formuler l'hypothèse d'une volonté d'éviter l'association avec l'héritage des Lumières, notamment avec la théorie de Rousseau (ce qui ne serait qu'une des facettes multiples de la relation complexe de Delille avec l'auteur des Confessions). De l'autre côté, la distinction entre la prononciation des voyelles et celle des consonnes a dégagé une logique profonde dans la division des sujets dans le troisième livre de La Conversation. L'analyse en termes de production et de perception du discours a rendu possible le relevé des sens variables pris sous la plume du poète par les mots «ton» et «accent», tout en mettant en évidence la répétition, avec une inflexion différente, du même tour «appelle la parole». Elle a permis également d'associer les passages aussi éloignés l'un de l'autre que les portraits des discoureurs dans La Conversation et l'image d'un exilé français retrouvant soudainement les sonorités de sa langue maternelle dans La Pitié ; enfin, elle a invité à percevoir le parallélisme entre le projet poétique de Delille et l'approche holistique, adoptée par 
les thérapeutes de son époque. C'est à la tradition scientifique de l'anatomie comparée que l'on peut rattacher aussi le penchant à envisager la parole en parallèle avec l'expression animale. Explicite dans Les Trois Règnes..., la comparaison apparait, sous la forme masquée souvent par la métaphore, dans La Conversation, où le mauvais discoureur est représenté sous les traits de l'animal («cheval», « oiseau », « chapon » [C, p. 55, 58 et 71]).

Deuxièmement, dans les textes étudiés on remarque l'application de la perspective double, suggérée par la division de la médecine en physiologie et pathologie. Dans La Conversation, à côté de la réflexion sur la prononciation normale, nous retrouvons la description d'un discours entravé. Dans La Pitié, la douce voix entretenant le lien social s'oppose aux cri accompagnant l'exécution du souverain $(P$, p. 97). Peut-on extrapoler, en demandant si la présentation des anti-modèles du comportement ne rattache pas la veine préscientifique des Caractères à la galerie des types pathologiques modernes, décrits entre autres dans les ouvrages de Gall? S'il serait excessif d'adopter cette conclusion, il n'en est pas moins vrai que Delille appartenait à une époque qui réfléchissait profondément sur la relation entre norme et pathologie. Dans La Pitié, la révolution est présentée tantôt comme conséquence explicable des abus sociaux, tantôt comme violation des règles de la nature $(P$, p. 126). Dans son grand ouvrage d'interrogation de la frontière entre le physiologique et le pathologique, Georges Canguilhem fait un usage (lui-même devenu célèbre) de la phrase de Leriche : «La santé est la vie dans le silence des organes» (1966, p. 52). Cette métaphore, fondée sur l'analogie avec la voix, s'applique curieusement à la théorie hautement normative de la conversation, proposée par Delille : les interlocuteurs « sains » sont ceux qui savent se taire pour laisser parler l'autre.

Dans ce contexte, la gravure de Nicolas Antoine Taunay représentant l'auteur en train de déclamer son poème au salon de Mme Geoffrin $(C$, avant la p. 131) fait figure d'éloge de l'équilibre entre le silence et la parole, inscrit au cœur de l'idéal delillien de la conversation. Tout en offrant aux yeux du spectateur le poète parlant devant un groupe de gens silencieux, l'illustrateur nous montre non seulement un auditoire animé (comme il pouvait l'être lors de la récitation d'une scène tragique, par exemple), mais surtout un auditoire s'adonnant à une série d'actes de communication muets. Mme Geoffrin, à qui un homme assis derrière offre un mouchoir, lève sa main. Son geste n'est pas toutefois sans évoquer le mouvement tranchant dont on appuie d'habitude une réplique pleine d'énergie et de gravité. Les yeux baissés, un autre homme se penche dans son siège. Est-il plongé dans la méditation ou distrait par l'action soudaine ? Cherche-t-il le regard d'un homme assis derrière Delille ? Nous voyons les gens " [se] comprendre du geste et [se] parler des yeux » $(P$, p. 103). Tout en imposant le silence à l'entourage, la récitation fournit l'occasion à un échange des idées, même si - du côté de l'auditoire - celui-ci doit se passer de voix. La santé de la conversation est la communication - y compris celle qui s'accompagne du silence des organes de la parole. 


\section{BIBLIOGRAPHIE}

Anonyme (1765). Voix. In Encyclopédie Dictionnaire raisonné des sciences, des arts et des métiers. T. 11. Paris Neuchâtel : Samuel Faulche.

Canguilhem, G. (1966). Le Normal et le Pathologique. Paris : PUF.

Castil-Blaze (1820). De l'opéra en France. T. 1. Paris : Janet et Cotelle.

Clauzade, L. (2008). Le cerveau chez Cabanis et Gall : la philosophie biologique du XVIII ${ }^{\mathrm{e}}$ siècle en débat. In. C. Dupont \& C. Cherici (Éds.), Les Querelles du cerveau (pp. 235-255). Paris : Vuibert.

Colombat, M. (1838). Traité des maladies et de l'hygiène des organes de la voix. Paris : Mansut Fils.

Corsi, P. (2018). Systèmes de la nature and Theories of Life: Bridging the Eighteenth and Nineteenth Centuries. Republic of Letters, 6/1, 1-27.

Court de Gébelin, A. (1775). Le Monde primitif analysé et comparé avec le monde moderne considéré dans son génie allégorique et dans les allégories auxquelles conduisit ce génie. T. 3. Paris : Boudet, Valleyre etc.

Cuvier, G. (1805a). Leçons d'anatomie comparée. T. 2. Paris : Baudouin.

Cuvier, G. (1805b). Leçons d'anatomie comparée. T. 4. Paris : Baudouin.

Delille, J. (1803). La Pitié, poème. Paris : Giguet et Michaud.

Delille, J. (1808). Les Trois Règnes de la nature avec des notes par M. Cuvier, de l'Institut et autres savants. T. 2. Paris : Frères Mame.

Delille, J. (1812). La Conversation, poème en trois chants. Paris : L.G. Michaud. URL : https:// books.google.pl/books?id=F5xFAQAAIAAJ\&printsec=frontcover\&hl=pl\&source=gbs_ge_su mmary_r\&cad $=0 \# \mathrm{v}=$ onepage $\& \mathrm{q} \& \mathrm{f}=$ false

Du Marsais, C. Ch. (1751). Accent. In Encyclopédie Dictionnaire raisonné des sciences, des arts et des métiers. T. 1. Paris : Briasson etc.

Dumas, Ch.-L. (1800a). Principes de physiologie, ou Introduction à la science expérimentale, philosophique et médicale de l'homme vivant. T. 2. Paris : Crapelet.

Dumas, Ch.-L. (1800b). Principes de physiologie, ou Introduction à la science expérimentale, philosophique et médicale de l'homme vivant. T. 3. Paris : Crapelet.

Gérando, J.-M. de (1799). Des Signes et de l'Art de penser considérés dans leurs rapports mutuels. T. 1. Paris : Goujon.

Gineste, T. (1989). La pensée médico-psychologique de Jean-Marc Gaspard Itard. Bulletin de la Société d'histoire de la médecine, 23 (2), 115-120.

Guitton, É. (1976). Jacques Delille (1738-1813) et le poème de la nature en France de 1750 à 1820. Lille : Service de reproduction des thèses Université de Lille III.

Itard, J.-M. G. (1894). Rapports et mémoires sur le sauvage de l'Aveyron, l'idiotie et la surdimutité. Paris : F. Alcan.

Jamain, C. (2004). Idée de la voix. Études sur le lyrisme occidental. Rennes : Presses Universitaires de Rennes.

Jaucourt, L. de (1765). Oreille. In Encyclopédie Dictionnaire raisonné des sciences, des arts et des métiers. T. 11. Paris Neuchâtel : Samuel Faulche.

Louâpre, M. (2014). La poésie scientifique : autopsie d'un genre. M. Louâpre, H. Marchal \& M. Pierssens (Éds.), La Poésie scientifique de la gloire au déclin (pp. 21-42). URL: www. epistémocritique.org

Louâpre, M., Marchal, H. \& Pierssens, M. (Éds.). (2014). La Poésie scientifique de la gloire au déclin. URL : www. epistémocritique.org

Marchal, H. (2014). Les sciences peuvent avoir quelques obligations à la poésie: Delille et l'institution savante. Francofonia, 67, 91-106. URL : https ://www.jstor.org/stable/24808480 
Marchal, H. (2016). Delille plastique. In Ph. Auserve (Éd.), Jacques Delille l'oublié ? Table ronde du 8 novembre 2013 (pp. 71-96). Clermont-Ferrand: Académie des sciences, belles-lettres et arts de Clermont-Ferrand.

Marchal, H. \& Wanlin, N. (2014). Le prosimètre didactique et scientifique de la fin du XVIII ${ }^{\mathrm{e}}$ au début du $\mathrm{XX}^{\mathrm{e}}$ siècle. Atlantide, 1, 1-28. URL : http://atlantide.univ-nantes.fr/Le-prosimetredidactique

Mercier-Faivre, A.-M. (1999). Un supplément à l'Encyclopédie: le Monde primitif d'Antoine Court de Gébelin. Suivi d'une édition du Génie allégorique et symbolique de l'Antiquité, extrait du Monde primitif, 1773. Paris : H. Champion.

Moreau, J.-L. (1797-1798). Éloge de Félix Vicq-d'Azir. Paris : Laurens, Méquignon, Croullebois \& Desenne.

Neboit-Mombet, J. (2001). Évolution des connaissances sur la voix humaine de 1770 à 1870. In J. Wagner (Éd.), La Voix dans la culture et la littérature françaises 1713-1875 (pp. 24-34). Clermont-Ferrand : Presses universitaires Blaise Pascal.

Potton, A. (1843). Observations physiologiques et musicales. Analyse d'un mémoire des docteurs Diday et Pétrequin sur la voix sombrée. Lyon : L. Boitel.

Rousseau, J.-J. (1969). Essai sur l'origine des langues. Paris : Bibliothèque du Graphe.

Savary, A. Ch. (1812). Audition. In Dictionnaire des sciences médicales. T. II. Paris : Panckoucke.

Stalnaker, J. (2010). The unfinished Enlightenment : description in the age of the Encyclopedia. New York : Cornell University Press.

Steuckardt, A. \& Thorel, M. (Éds.) (2017). Le Jugement de l'oreille, XVI ${ }^{e}$-XVIII siècles.

Stewart, D. (1808). Élémens de la philosophie de l'esprit humain. T. 1. Trad. Pierre Prévost. Genève : Paschoud.

Viau, T. de (1941). Stances. In D. Aury, T. Maulnier (Éds.), Poètes précieux \& baroques du XVII . Angers : J. Petit.

Virgile (1804a). L'Enéide. T. I. Trad. J. Delille. In. J. Delille, Euvres. Paris : Giguet et Michaud.

Virgile (1804b). L'Enéide. T. II. Trad. J. Delille. In. J. Delille, Euvres. Paris : Giguet et Michaud.

Voisin, F. (1821). Du Bégaiement, ses causes, ses différens degrés, influence des passions, des sexes, des âges, etc. Paris : l'Auteur.

Wanlin, N. (2014). Le poème didactique: un genre réactionnaire ? La Révolution française. Cahiers de l'Institut d'histoire de la Révolution française, 7, 1-10. DOI : 10.4000/lrf.1198

Williams, E.A. (1994). Physical and the moral : Anthropology, Physiology, and Philosophical Medicine in France 1750-1850. Cambridge : Cambridge University Press. 